\title{
Photo-Chromeleon: Re-Programmable Multi-Color Textures Using Photochromic Dyes
}

\author{
Yuhua Jin*, Isabel Qamar*, Michael Wessely*, \\ Aradhana Adhikari, Katarina Bulovic, Parinya Punpongsanon, Stefanie Mueller \\ MIT CSAIL, Cambridge, MA, USA \\ \{yuhuajin, ipsqamar, wessely, adhikara, kbulovic, parinya, stefanie.mueller\}@mit.edu \\ *equal contribution
}

\begin{abstract}
In this paper, we present a method to create re-programmable multi-color textures that are made from a single material only. The key idea builds on the use of photochromic inks that can switch their appearance from transparent to colored when exposed to light of a certain wavelength. By mixing cyan, magenta, and yellow (CMY) photochromic dyes into a single solution and leveraging the different absorption spectra of each dye, we can control each color channel in the solution separately. Our approach can transform single-material fabrication techniques, such as coating, into high-resolution multi-color processes.
\end{abstract}

We discuss the material mixing procedure, modifications to the light source, and the algorithm to control each color channel. We then show the results from an experiment in which we evaluated the available color space and the resolution of our textures. Finally, we demonstrate our user interface that allows users to transfer virtual textures onto physical objects and show a range of application examples.

\section{Author Keywords}

personal fabrication; programmable matter; multi-color textures; color change; photochromic.

\section{CSS Concepts}

- Human-centered computing Displays and imagers

\section{INTRODUCTION}

Programmable matter that has the ability to change its physical properties (color, shape, density) holds the promise of a future in which objects will re-configure themselves according to a user's needs [5]. One aspect of programmable matter is color, which would allow objects to change their appearance repeatedly. For instance, in clothing, accessories could be altered to match the main outfit and textiles could be recolored for different events in the same day.

Permission to make digital or hard copies of all or part of this work for personal or classroom use is granted without fee provided that copies are not made or distributed for profit or commercial advantage and that copies bear this notice and the full citation on the first page. Copyrights for components of this work owned by others than ACM must be honored. Abstracting with credit is permitted. To copy otherwise, or republish, to post on servers or to redistribute to lists, requires prior specific permission and/or a fee. Request permissions from Permissions@acm.org.

UIST '19, October 20-23, 2019, New Orleans, LA, USA

(C) 2019 Association for Computing Machinery.

ACM ISBN 978-1-4503-6816-2/19/10 ..\$15.00

https://doi.org/10.1145/3332165.3347905
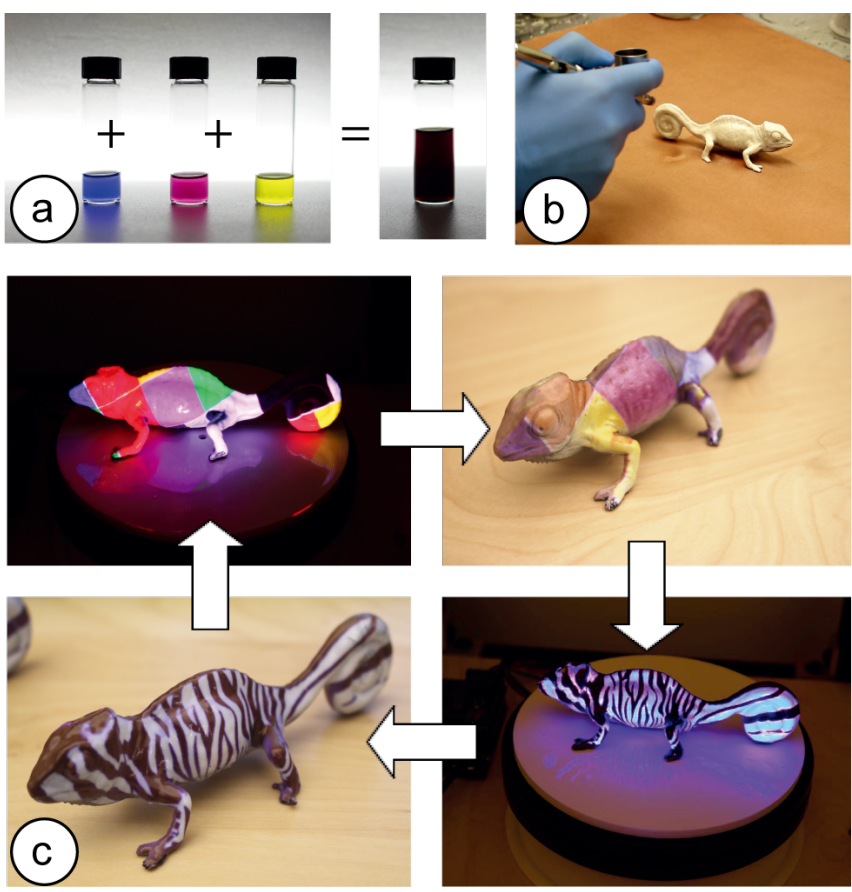

Figure 1. We can create re-programmable multi-color textures from a single material. (a) We mixed CMY photochromic dyes together to create our multi-color ink. (b) After coating the object, we use (c) a UV light source and a projector to control each color channel on a pixel-by-pixel basis, resulting in high-resolution multicolor textures that can be reapplied multiple times.

To update the appearance of objects, researchers started to use re-programmable materials, such as photochromic inks [4], that can switch from transparent to colored when exposed to light of a certain wavelength. Since the inks are bi-stable, the color remains even when the light source is removed. The process is fully reversible, therefore enabling users to recolor the object as many times as they desire.

A major limitation of using photochromic materials, however, is that they are single-color only, i.e. each material can only transition from transparent to one color and back to transparent (e.g. Photochromic Carpet [17]). To bypass this limitation, researchers printed a voxel pattern with one photochromic color per voxel across the surface of an object and then selectively deactivated all voxels of the colors that were 
not part of the desired appearance (ColorMod [15]). However, this approach had several limitations: (1) it was low resolution (1mm x $1 \mathrm{~mm}$ voxels) since each voxel had to be 3D printed, (2) it was limited to a few discrete colors only since each color had to be loaded as a separate 3D printing material, (3) it required a specialized $3 D$ printer with $4+$ print heads (one print head per color + infill).

Inspired by work from Hirayama et al. [9], we present an approach that addresses all three problems. By mixing cyan, magenta and yellow photochromic dyes into a single solution and leveraging the different absorption spectra of each dye, we can control each color channel in the solution separately, which results in a range of colors across the CMY color space. Since our approach uses only a single solution, we can transform single-material fabrication processes, such as coating, into high-resolution multi-color techniques (Figure 1).

The benefits of our approach are as follows: (1) since we no longer need to print individual voxels for each color, we can create high-resolution textures that are limited only by the precision of the projector; (2) since we can control each color channel and can determine to which extent it should be deactivated, we can create a range of intermediate colors in the CMY color spectrum and are no longer limited to a few discrete colors; (3) since we only need a single material for our approach, it eliminates the need for specialized 3D printing hardware and allows us to use simple fabrication techniques, such as spraying or coating instead. Note that we do not claim to achieve the entire CMY color spectrum with our method due to partial deactivation across channels (see Figure 14 for the available color space).

We begin by summarizing the related work on photochromic systems and then introduce the main working principle of our approach. We then describe how we developed the CMY photochromic solution, how we modified the projector to output the correct wavelengths for deactivating the different photochromic dyes, and how we determined the achievable color gamut through our evaluation. Finally, we present a range of scenarios that demonstrate our system in practice.

\section{RELATED WORK}

Our work is related to research in reprogrammable textures via photochromics in HCI and computer graphics. A review of photochromics from the perspective of material science can be found in Ferrara et al. [4].

\section{Single Color Photochromic Systems}

The majority of HCI projects that use photochromic materials have focused on single color systems since individual colors are commercially available. For instance, Photochromic Carpet [17] turns into a colored state capturing foot prints when users walk with shoes that have embedded UV LEDS in the sole. Similarly, users can 'draw' onto a Photochromic Canvas [6] using a hand-held UV projector, which is used to transfer computer generated textures onto the canvas. Other systems use photochromic paper for telecommunication, i.e. computer-controlled UV LEDs use the photochromic paper as an output medium for transferring information (KAMI CHAT [14]). Instead of using UV LEDs or projectors, SolaColor [8] uses the sun for activation, i.e. its outdoor display spatially varies the content with movements of the sun.

Similar single-color systems also exist for other types of chromogenic materials, such as thermochromics (activated by heat rather than light). Applications range from interactive touch screens (Inkantatory Paper [20]), fashion (Shimmering Flower [1], ShaderPrinter [16]) and on-body interfaces, such as tattoos (AnimSkin [21], DuoSkin [13]) and makeup (ChromoSkin [12]).

\section{Multi-Color Photochromic Systems Based on Pixels}

To create systems capable of producing more than one color, researchers have investigated how to use a pattern of differently colored photochromic pixels. Photochromic Sculpture [7] uses a stack of transparent 2D sheets, each of which contains a pattern of painted photochromic pixels in different colors. To allow the light to reach each pixel, however, a large offset is necessary between the pixels on each sheet. In addition, painting the dyes across the object did not scale to more complex 3D geometries. To address these issues, ColorMod [15] extended the work by developing 3D printable photochromic inks that enabled the creation of a photochromic voxel pattern across the surface of an object. The limitations of this approach included the manufacturing resolution ( $1 \times 1 \mathrm{~mm}$ voxels), color gamut (max. 3 colors), and required complex fabrication hardware (custom 3D printer with 4+ printheads [18]).

\section{Multi-Color Systems Based on Blending}

Another method researchers explored to create a larger color gamut is to stack multiple single-color layers and selectively blend them together. The Paint Color Control System [23], for instance, uses a layer of blue thermochromic ink (activated/deactivated by heat) on top of regular yellow ink. When heat is applied, the blue thermochromic ink transitions from transparent to colored, thereby blending in with the yellow ink, resulting in different shades of green. The limitation of this approach is that only the top most layer can be activated or deactivated, thereby allowing for only one controllable color channel. Anabiosis [19] furthers this approach by creating a stack of sheets containing regular and thermochromic ink: When heat is supplied to the stack by human touch, an increasing number of layers become visible as the heat propagates through, leading to a composite image made up from all the visible ink layers. This method, however, is limited in that the image has to be pre-computed.

Closest to our work is an approach presented by Hirayama et al. [9], which explores how to mix multiple photochromic inks into a single solution. While they follow the same goal as our research, the paper presents only one working example of two color channels: cyan and yellow (resulting in green). We show in this paper that achieving all three channels: cyan, magenta, and yellow, is challenging since magenta partially overlaps with cyan and yellow in the deactivation spectrum, 
thus requiring modifications to the light source and a computational method to find an optimal sequence of deactivation wavelengths for a pixel to take on a desired color. In addition, no end-to-end system was presented that would allow users to transfer multi-color textures onto 3D objects.

\section{BASIC WORKING PRINCIPLE}

Photochromic dyes can transform from a transparent to a colored state through the absorption of UV light ('activation'), and transform back from colored to transparent through the absorption of visible light ('deactivation') [22].

When cyan, magenta, and yellow photochromic colors are mixed together into a single solution and the solution is activated with UV light (i.e. all three color channels are fully saturated), the resulting color is black (Figure 2a). This is consistent with the CMY color chart shown in Figure 2b, i.e. the center of the chart, which shows the result of all three color channels in full saturation is black.

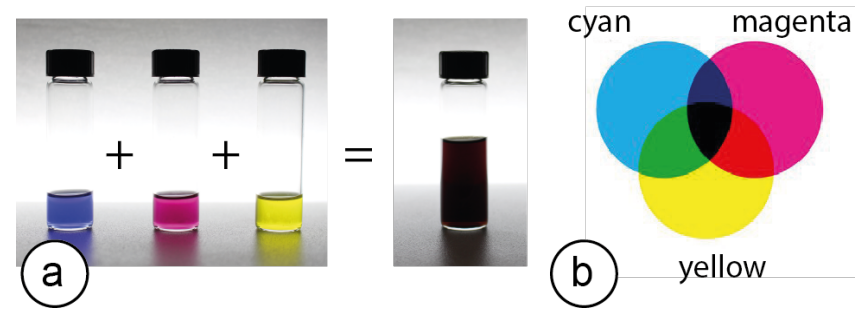

Figure 2. (a) CMY inks mixed together achieve black, which matches (b) the CMY color model.

To achieve colors other than black, we need to deactivate one or more color channels. Deactivating the cyan color, for instance, would result in red since only yellow and magenta remain activated (Figure $2 \mathrm{~b}$ ). To deactivate each color channel individually, we can leverage the fact that the deactivation peak (i.e. absorption peak) of each photochromic color is at a different wavelength (Figure 3).

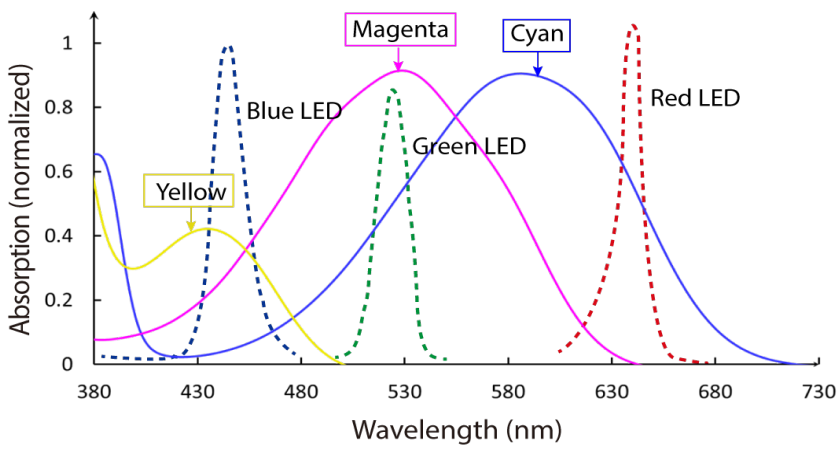

Figure 3. The absorption peak of each photochromic dye is at a different wavelength. To control each color channel, we can use the RGB LEDs of a projector to supply the required deactivation wavelength.

Since all of the deactivation wavelengths are within the spectrum of visible light (390nm to 790nm), we can use a regular office projector's red, green, and blue LEDs to supply one deactivation wavelength each. As can be seen in Figure 3, shining blue light from the projector will deactivate yellow, green light will deactivate magenta and red light will deactivate cyan. Thus, to deactivate a specific photochromic color channel, the projector only needs to project $\mathrm{R}, \mathrm{G}$, or B pixels to reduce the saturation of the channel, as shown in Figure 4.

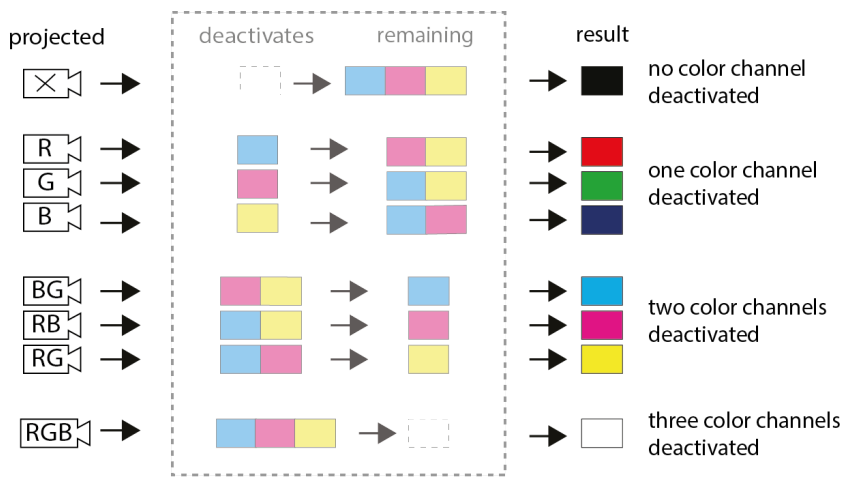

Figure 4. Theoretical result of shining different combinations of RGB on the mixed CMY photochromic ink.

Figure 5 shows the physical result of shining different combinations of RGB on the CMY ink mixture, which we obtained by following the deactivation chart in Figure 4. While Figure 5 demonstrates the results obtained by fully deactivating one or more channels, we can achieve intermediate colors of different saturation levels by only partially deactivating each channel. We will provide more information on this when we discuss the algorithm that times the projected deactivation wavelengths to accomplish this.

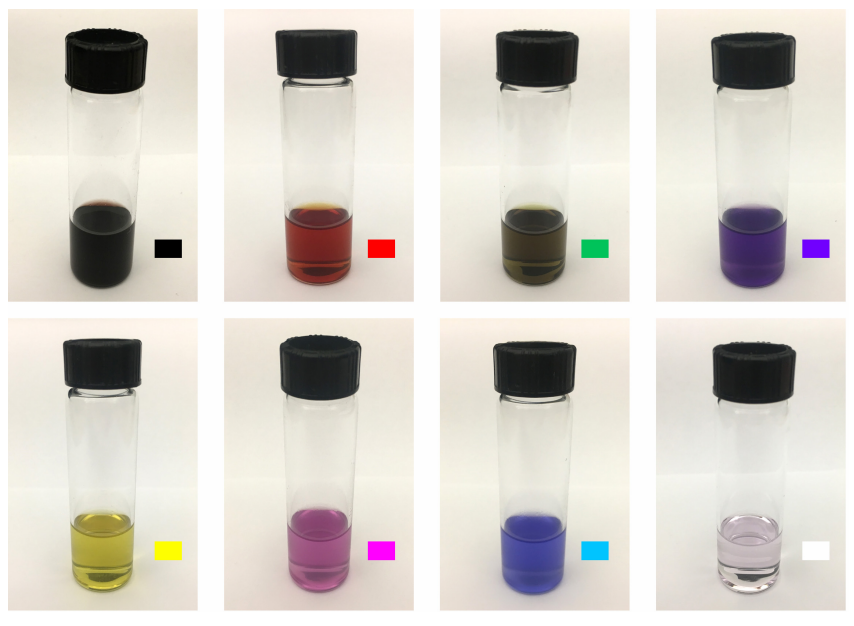

Figure 5. Physical result of shining different combinations of RGB on the mixed CMY dye.

In the remainder of this paper, we will provide more detail on each of the steps, starting with (1) creating the photochromic CMY coating that can be applied to objects, (2) modifying the light output from the projector to ensure the wavelength of the light matches with the deactivation wavelength of the dyes, and (3) developing the algorithm to accomplish intermediate colors of different saturation levels. After this, we will (4) evaluate the color gamut that we can achieve with our method and determine how closely the 
physical color represents the virtual color. Finally, we will (5) discuss the implementation of our end-to-end system to transfer the texture onto an object.

\section{DEVELOPING THE PHOTOCHROMIC COATING}

To make our method directly applicable to physical objects, we developed a re-programmable photochromic coating that can be airbrushed onto the surface of objects. To develop the coating, we dissolved cyan, magenta and yellow photochromic dyes directly into a transparent laquer.

\section{Creating the ink mixture}

Several factors were involved in selecting the dyes for the CMY solution: (1) The visual appearance of the dyes needs to be as close to cyan, magenta, and yellow as possible in order to achieve the largest color gamut; (2) to be able to control each color channel individually, the deactivation wavelengths for each photochromic dye need to have as little overlap as possible; (3) the color of the dyes needs to be stable (i.e. do not deactivate quickly under ambient light).

\#1 Visual appearance: Figure 6 shows the available dyes from Yamada Chemical Co. [22] which is the only company we found that reliably sells bi-stable (P-type) photochromic dyes. While we can see that a dye color exists for yellow (DAE-0068); for magenta, the nearest colors are red purple (DAE-0012), red (DAE-0004) and purple (DAE-0159), and for cyan: blue (DAE-0001) and blue purple (DAE-0018).

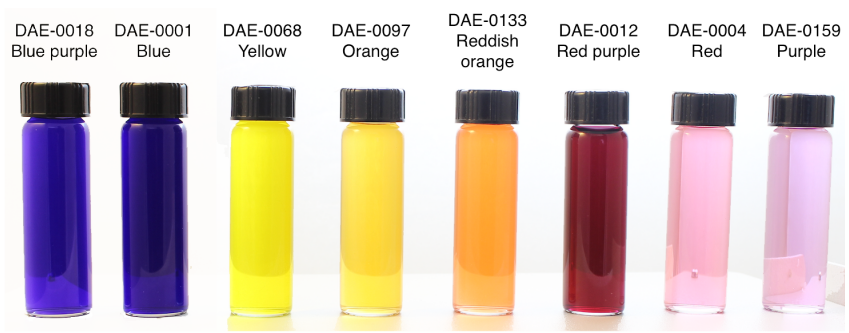

Figure 6. Bi-stable photochromic inks available from Yamada Chemical Co. [22]: $0.1 w t \%$ die mixed in ethyl acetate.

\#2 Absorption Spectra: To be able to choose photochromic dyes that minimize overlap between different color channels, we determined the absorption spectrum for each photochromic dye. For this, we first mixed $0.1 \mathrm{wt} \%$ of each dye in ethyl acetate from Fisher Scientific [11] using a magnetic stirrer for 1 hour at 500 RPM and then filled the solution into quartz cuvettes with a $1 \mathrm{~mL}$ path length. We then radiated the solution under UV light until the photochromic inks were fully activated and then placed the cuvettes in a spectrophotometer (Varian Cary 5000 UV-Vis-NIR spectrophotometer) to determine their absorption spectra. The results are shown in Figure 7. We used yellow (DAE-0068) as a starting point for selection since it was the only available dye for this channel. For magenta, red purple (DAE-0012) and red (DAE004) had the least overlap with yellow and cyan. For cyan, the least overlap with magenta was blue (DAE-0001) followed by blue purple (DAE-0018).

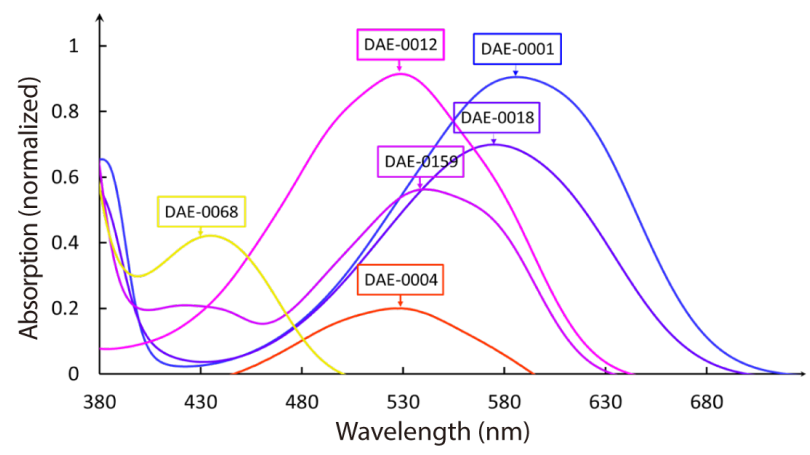

Figure 7. Absorption spectra of the photochromic dyes in the visible wavelength range.

\#3 Stable under ambient light: Both red (DAE-0004) and blue purple (DAE-0018) were not very stable as they deactivated in ambient light after a few minutes. Thus, based on our criteria, we decided to use for cyan: blue (DAE-0001), for magenta: red purple (DAE-0012), and for yellow: yellow (DAE-0068). This combination minimized overlap of deactivation wavelengths while also being closest to the desired color channels and the greatest stability under ambient light. For the purpose of easier explanation, we will refer to the blue dye as cyan dye and the red purple dye as magenta dye for the remainder of the paper.

\section{Applying the ink to materials \& objects}

Before mixing the three photochromic dyes together, we first mixed each dye separately in laquer (Dupli-Color Paint Shop Finish Systems Matte-Finish Clear Coat (BSP307) [3]). For the three separate mixtures, we used $0.05 \mathrm{wt} \%$ cyan, $0.05 \mathrm{wt} \%$ magenta and $0.3 \mathrm{wt} \%$ yellow respectively. These concentrations were chosen based on the deactivation times of each dye: Because yellow deactivates faster than the other two dyes, the concentration of the yellow dye was increased in order to extend the illumination time required for this dye to deactivate. We then mixed the resulting liquids by equal volumes $(1: 1: 1)$ to achieve our multi-color coating.

Before applying the coating to an object, we primed the surface of the object with spray paint to avoid subsurface scattering of the projected light. We first sprayed each object with black paint (drying time: $30 \mathrm{~min}$ ), and then subsequently sprayed a white paint layer (drying time: 24 hours).

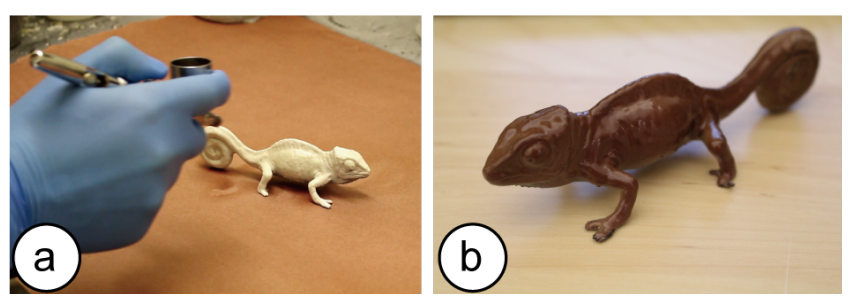

Figure 8. (a) Applying the photochromic coating and (b) the resulting coated object after UV activation.

After this, we sprayed our photochromic coating onto the surface of the object using an airbrush system (Iwata HP$\mathrm{CS}$ ). The saturation of the sprayed ink can be increased by 
applying a second layer of ink after letting the first layer dry for 20 minutes. The ink is then fully dried after ca. 24 hours.

By using an airbrush to apply the coating, we can coat the surface of an object evenly. While we sprayed the layers manually and found the consistency to be good enough for our applications, a CNC airbrush system may further improve the results. The coating can also be painted, however, this leads to an appearance that is less uniform.

We used laquer as the carrier material for the photochromic dyes. The laquer is recommended for use on metal (see example of model car exterior in Figure 25) and plastic (3D printed chameleon in Figure 8).

\section{MODIFYING THE PROJECTOR}

As mentioned previously, to deactivate each photochromic color channel individually, we need three different light sources: one light source with the corresponding deactivation wavelength for each photochromic dye. Since existing DLP projectors already contain three light sources (3 LEDs for $\mathrm{R}, \mathrm{G}, \mathrm{B})$, we decided to modify an existing office projector (model: AAXA M6, 1200 Lumens).

To determine the optical spectrum of each of the LEDs in the projector, we used a spectrometer (Thorlabs, CCS200) and found that the three LEDs overlap well with the selected photochromic dyes (Figure 9).

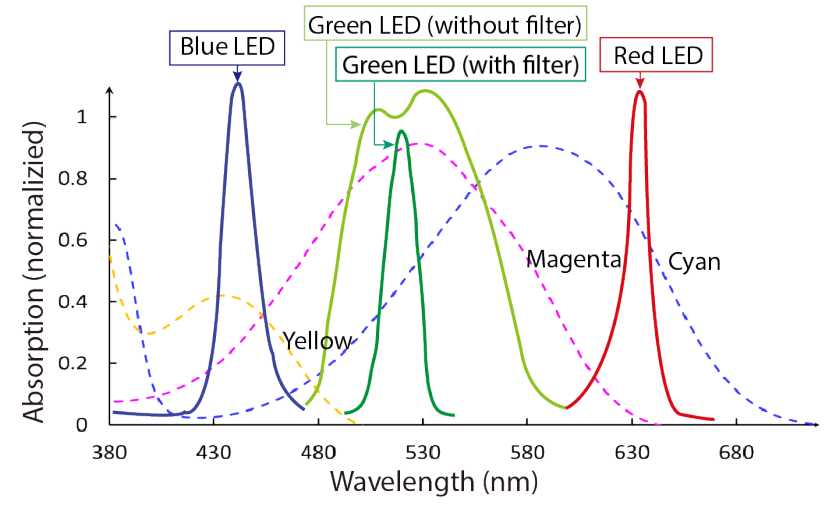

Figure 9. Optical spectrum of the projector output and absorption spectra of cyan, magenta and yellow dyes.

The blue LED of the projector creates light output over a small wavelength range and matches well with the yellow deactivation wavelength, therefore, by shining blue light from the projector, we can deactivate the yellow photochromic. Similarly, the red LED of the projector creates a light output over a small wavelength range and matches well with the cyan deactivation wavelength, thus, by shining red light, we can deactivate the cyan photochromic color.

Unfortunately, while the green LED of the projector matches well with the magenta deactivation wavelength, the default green LED creates a light output over a broader wavelength range. As a result, while shining green light would deactivate the magenta dye, it would also deactivate large parts of the yellow and cyan dyes.
To limit the wavelength range to only deactivate magenta, we opened up the projector and added a filter in front of the green LED (Semrock Brightline ${ }^{\circledR}$ FF02-529/24-25). To ensure the highest energy output, we placed the filter between the green LED and the collimator (Figure 10). With this modification, we were able to otherwise use the projector as is.

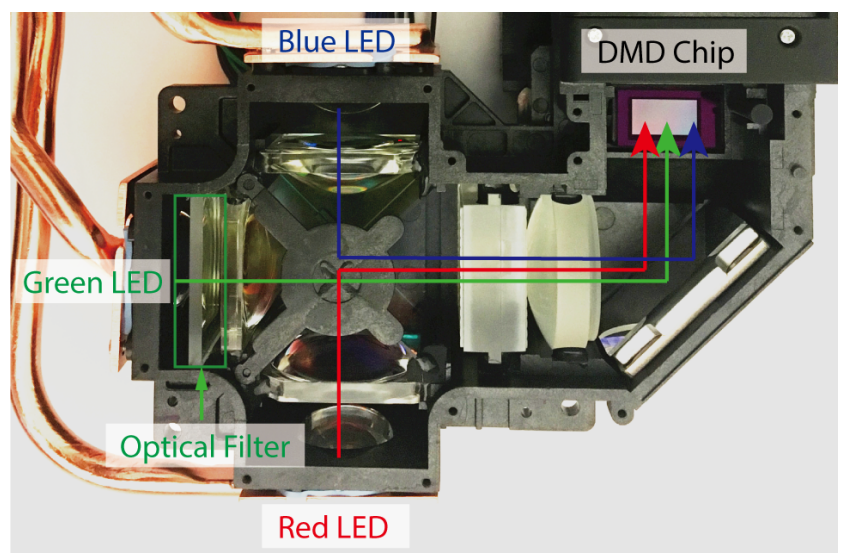

Figure 10. Modified projector with added optical filter between green LED and collimator.

\section{ALGORITHM TO COMPUTE DEACTIVATION TIMES}

With the modified projector at hand, we can now create a projection image to control the photochromic color channels of each pixel on the physical object.

\section{Effect of Projector Distance on Color Gamut}

When starting our research, we had initially placed the photochromic mixture ink directly in front of the projector, i.e. only a few centimeters away from the lens, which produced the results shown in Figure 5. We found that once we placed the photochromics further away from the projector, i.e. at a projection distance of $30 \mathrm{~cm}$ to allow the projected image to be in focus, the available color gamut reduced. Figure 11 shows the result acquired when projecting the same R,G,B sequence as in Figure 5 for comparison. Our assumption is that this difference is due to the change in light intensity, which we will explore in more detail in future work. By determining a relationship between projector distance and deactivation time, this could enable us to add 'projector distance' as a variable into our system to accommodate different placements of the objects to be re-colored.

\section{Naïve approach vs. Optimization algorithm}

In an ideal scenario each LED would deactivate only one color channel, however, as seen in Figure 9, shining light from one of the LEDs also partially deactivates the other two color channels. This has two implications: First, using the photochromic dyes commercially available today, we can achieve only part of the CMY color spectrum (Figure 14). For instance, it is not possible to achieve a fully saturated cyan as deactivating magenta also causes cyan to partially deactivate. Second, a naïve approach that assumes that each projected deactivation wavelength $(R, G, B)$, only affects a single photochromic color channel (Figure 4) will not lead to the correct color on the object due to additional effects of the 
other color channels on the dye. We therefore developed an optimization algorithm that takes into account the effect of overlapping absorption spectra. Figure 11 shows (a) the desired color texture and the results from both (b) the naïve approach and (c) our approximation algorithm, the latter of which leads to a color representation closer to the desired.
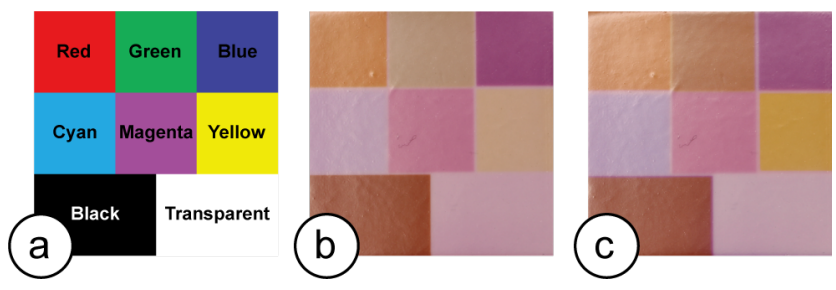

Figure 11. (a) Expected result. Results from (b) the naïve approach and (c) our optimization.

\section{Deriving the Parameters of the Algorithm}

Since our algorithm takes into account the effect each projector LED has on each photochromic color channel (i.e. its target photochromic color channel and the side effects on the other two channels), we first had to determine saturation decrease over time for each combination of LED and dye.

Apparatus and Procedure: To capture the deactivation times of each dye when exposed to each of the wavelengths R, G, $\mathrm{B}$ from the projector, we airbrushed cyan, magenta, and yellow coatings onto seperate white cubes. We placed each cube in front of the projector and fully activated the photochromic dye by shining UV light on its surface for 30 s.

We then shone all three deactivation wavelengths red (R), green $(\mathrm{G})$ and blue $(\mathrm{B})$ onto each cube in order to quantify the effect of the projector's LEDs on each dye.

The saturation level of each projected R, G, B color bar linearly increased from left to right over time until it reached the right edge of the cube (Figure 12). This procedure created a color gradient from fully saturated dye (left) to increasingly desaturated dye (right) and intermediate saturations in between. We then extracted the relative saturation level by capturing a photo of each cube under white light, converted the photo to the CMY color space, and plotted the saturation per color channel as the relative saturation decrease over time.

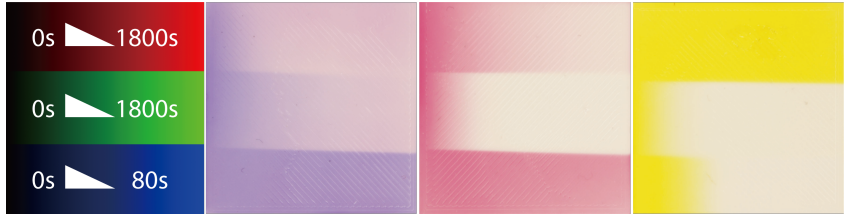

Figure 12. Effect of exposure time of $R, G, B$ projector channels on $C, M, Y$ coatings (left $=0 \mathrm{~s}$, with time increasing to the right).

Result: Figure 13 shows the relative saturation levels over time for each dye and each of the projector's color channels. While each of the projector's R, G, B LEDs deactivated its primary photochromic color channel, the deactivation times varied significantly from 32 seconds for the yellow dye under blue light to 620 seconds for the magenta dye under green light and 800 seconds for the cyan dye under red light.

Computing Deactivation Times: We used the data on relative saturation level over time as input into our approximation algorithm. To minimize the global error rate across all three channels, our algorithm proceeds as follows: Let $t=$ $\left(t_{r}, t_{g}, t_{b}\right)$ be the illumination time of the projector's color channels, then the estimated color of a coated surface $C(t)$ can be written as:

$$
C_{j}(t)=X-a_{j} t_{r}-b_{j} t_{g}-c_{j} t_{b} \quad j \in c, m, y
$$

Where $X \in C, M, Y$ are cyan, magenta, yellow at full saturation and $a_{j}, b_{j}, c_{j}$ are linear factors on the saturation reduction in relation to the illumination time $t$ per color channel.

Let $P$ be the target color, then we want to minimize the expression:

$$
\min F(t)=|C(t)-P|^{2}
$$

We use a gradient descent scheme which can be written as:

$$
t_{i+1}=t_{i}+\gamma\left(\begin{array}{lll}
a_{c} & a_{m} & a_{y} \\
b_{c} & b_{m} & b_{y} \\
c_{c} & c_{m} & c_{y}
\end{array}\right)\left(\begin{array}{l}
F_{r}(t) \\
F_{g}(t) \\
F_{b}(t)
\end{array}\right)
$$

With $\gamma$ being the step size.

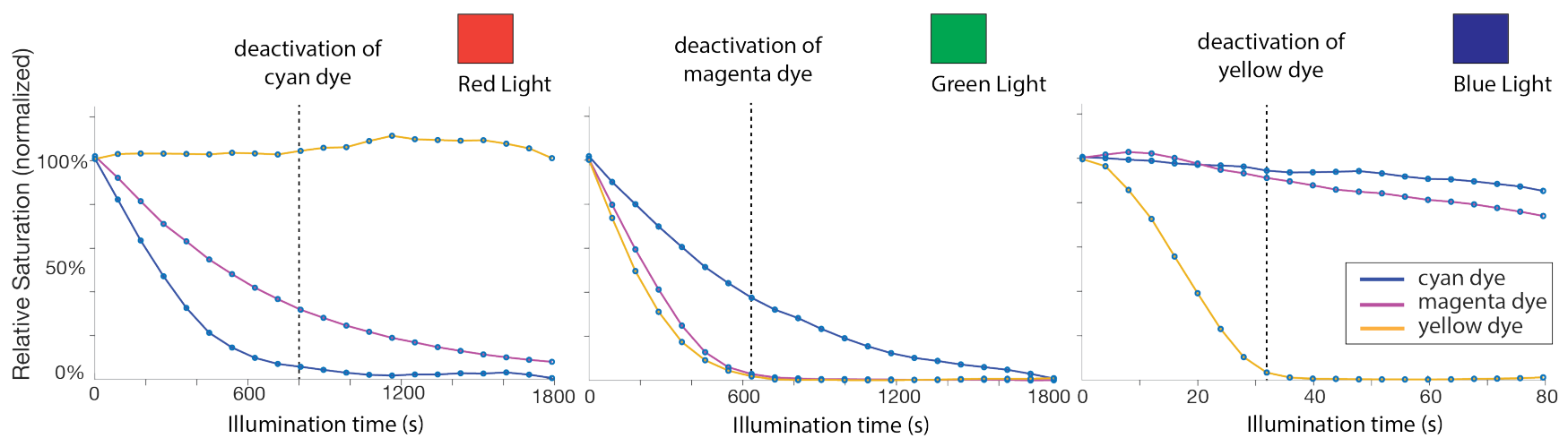

Figure 13. Deactivation times of the photochromic dye per light channel $(R, G, B)$. We consider a dye to be deactivated when it drops below $5 \%$ saturation. 
An example of the improvement of our optimization algorithm over the naïve approach was shown in Figure 11. Our greatest enhancement is in the yellow color, a result of our optimization algorithm, which prioritizes the red LED over a combination of the red and green LED (as predicted in Figure 4) to deactivate the cyan and magenta dyes (since the green LED also deactivates the yellow dye).

\section{EVALUATING OUR SYSTEM}

To evaluate our approach, we measured the available color gamut and the maximum resolution of the projected textures.

\section{Available Color Gamut}

First, we were interested in the color gamut we could achieve with the photochromic dyes purchased from Yamada Chemicals. To determine this, we placed a white cube coated with the CMY solution and fully activated it with the UV light until the coating appeared black. To sample the available color gamut, we took 5 images at evenly spaced deactivation times across the maximum deactivation length, i.e. for red/green: 1800 s and blue: $45 \mathrm{~s}$. This created $5 \times 5 \times 5$ sample images. After each light exposure, we used a camera to capture an image of the resulting outcome. We then converted the image into the CMY color space and extracted the mean value of each color channel using an OpenCV script.

Figure 14 shows the captured texture colors of this experiment in the CIExy chromaticity diagram. As can be seen, our color gamut has its greatest impact in the area between the three primary photochromic colors.

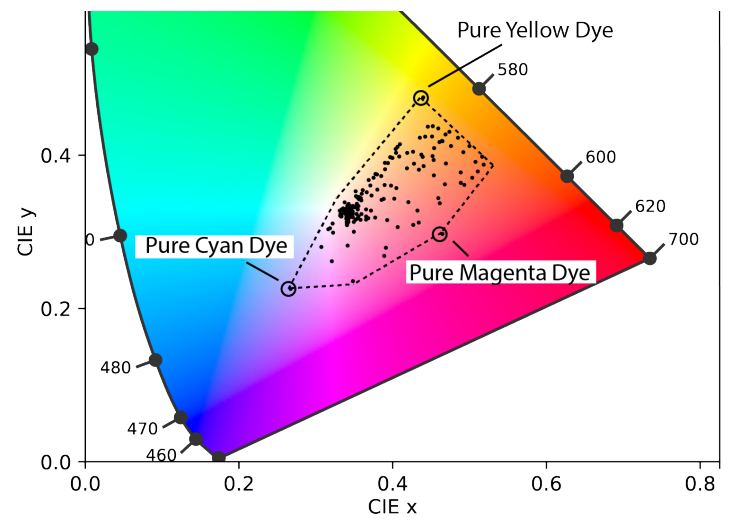

Figure 14: Achievable color gamut of our photochromic coating in the CIE xy chromaticity diagram.

\section{Texture Resolution}

Next, we quantified the texture resolution that can be achieved using the photochromic coating. We projected three black and white checkerboard patterns with varying checkerboard widths (5px, 2px, and 1px width), onto the surface of a coated cube and applied the texture on the cube for 12 minutes until the white checkerboard areas (white $=$ all three $\mathrm{R}, \mathrm{G}, \mathrm{B}$ LEDs on) were fully deactivated. We measured the pixel size of the physical texture using an Olympus SZ61 microscope (Figure 15) and found the pixel measurements to be consistent at $129 \mu \mathrm{m}$ for a single pixel $(5 \mathrm{px}=647 \mu \mathrm{m}, 35 \mathrm{px}=$ $257 \mu \mathrm{m}, 1 \mathrm{px}=129 \mu \mathrm{m})$.

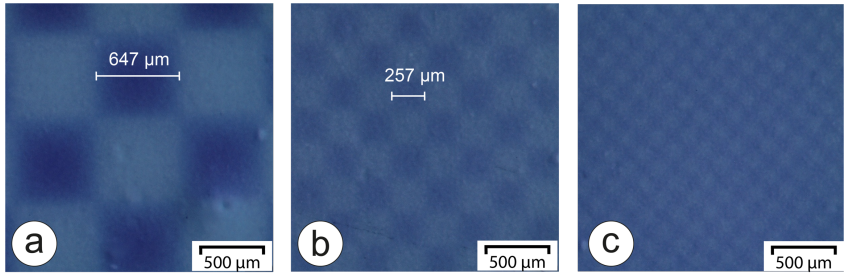

Figure 15: Resolution of checkerboard pattern.

While Figure $15 \mathrm{a} / \mathrm{b}$ (5px and $2 \mathrm{px}$ width) both show a clear checkerboard pattern, the squares start to become indistinguishable in Figure 15c (1px width). To clarify if the blur at the edges of the individual pixels in Figure 15 stems from the projector being out-of-focus or from subsurface scattering, we ran an additional test: we attached a $1 \times 1 \mathrm{~cm}$ square mask on a UV activated cube and projected white light on it, after which we removed the square mask. Examining under a microscope, the edges and corners of the pixels remained sharp indicating that the projector's lens and focus is the main reason for blur. Thus, while with our current projector system, the maximum resolution we can achieve is $257 \mu \mathrm{m}(2 \mathrm{px})$, a better projector could achieve higher texture resolutions.

\section{Color Fade Over Time}

The time for a texture to fade depends on the saturation level of the texture being applied to the object. A stronger saturation will take longer to deactivate fully than a lighter saturation. To evaluate the durability in normal lighting conditions, we activated three samples, coated with $\mathrm{C}, \mathrm{M}$, and $\mathrm{Y}$ colors, with a UV light and placed them under 150 lux illumination (average light intensity of a living room is 100-300 lux [1]). We recorded the time each color took to disappear: the cyan and magenta colors disappeared after $26 \mathrm{~h}$ and $19 \mathrm{~h}$ respectively, while the yellow color disappeared after $5 \mathrm{~h}$ (Figure 16). Since outside light is stronger than indoor lighting, the dyes deactivate quicker and are therefore more suitable for indoor use. These deactivation times are a limitation in the materials currently commercially available.

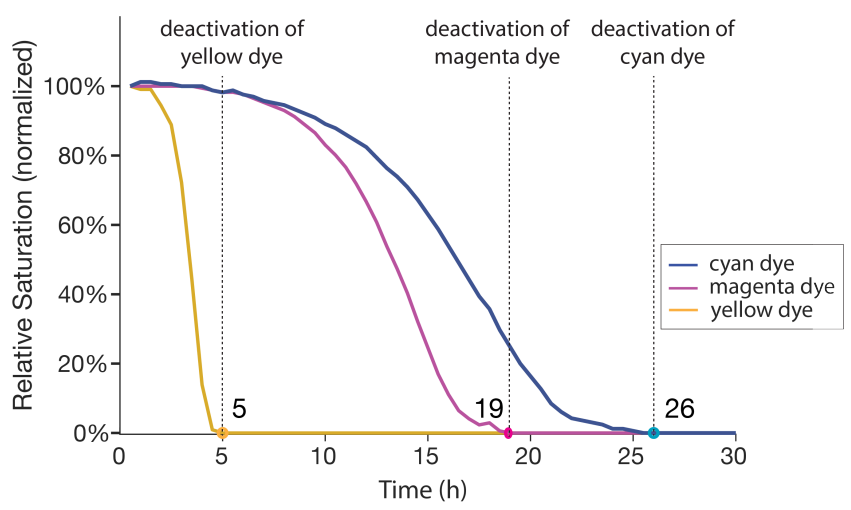

Figure 16. Color fade vs time for cyan, magenta and yellow dyes under 150 lux illumination. 


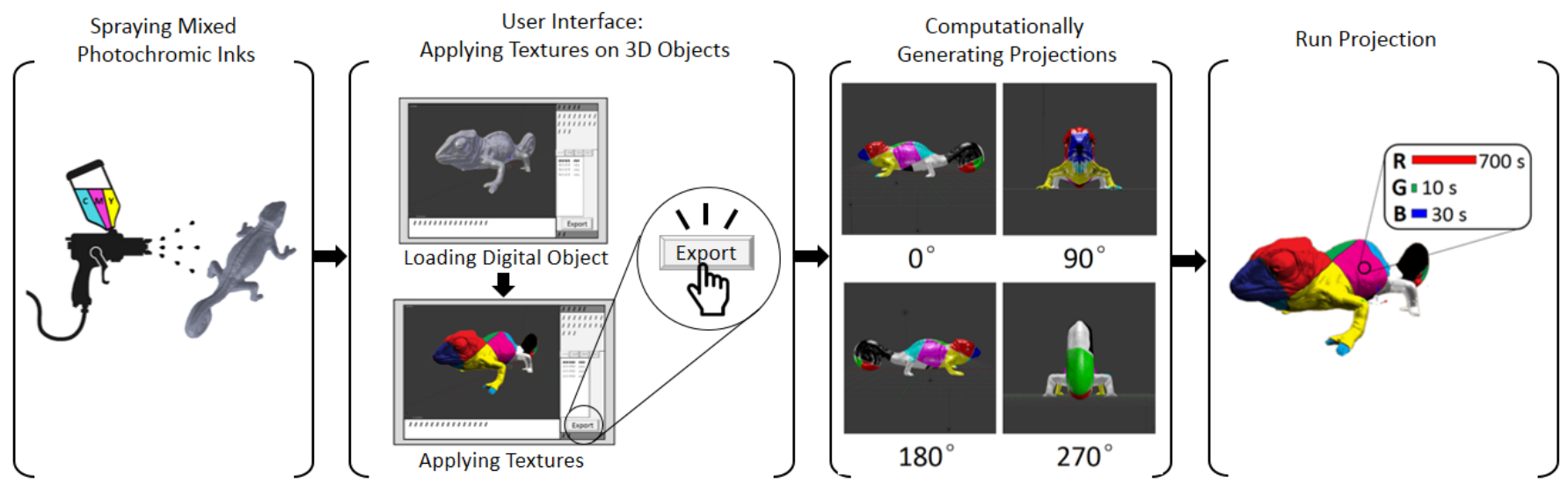

Figure 17. Overview of the end-to-end system.

\section{END-TO-END SYSTEM TO TRANSFER A TEXTURE}

In the last part of this paper, we describe the end-to-end system that allows users to transfer a texture onto a physical object. Figure 17 shows a summary of the system pipeline.

\section{Hardware Setup}

We use a similar hardware setup and component layout as ColorMod [15]: For activation, we use a UV light (Luminus Devices Inc. CBM-40-UV, $\sim 365 \mathrm{~nm}, 4 \mathrm{~W}$ ) that we automatically turn on/off using a digital controller (PhatLight LED Develop Kits). For deactivation, we use our modified projector (LED DLP projector AAXA M6, 1920x1080 pixels, 1200 Lumens, with added green filter). For $360^{\circ}$ projection on the object, we use a rotating platform that is controlled with a stepper motor. The rotating platform has a positioning screw to ensure central placement of the object when rotating. The setup with the above components is shown in Figure 18.

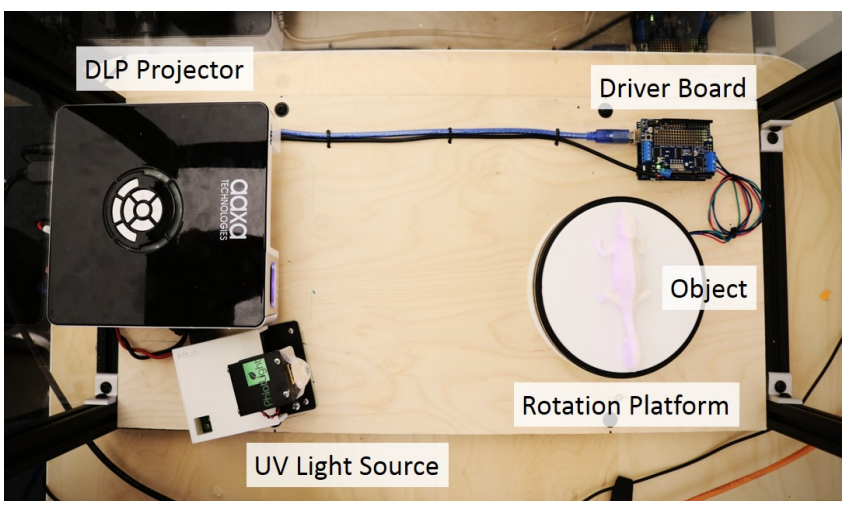

Figure 18. Hardware setup.

\#1 Applying a Virtual Texture to a 3D Model

After placing the physical object onto the rotating platform, users load the corresponding 3D model into the 3D editor Blender (Figure 19a). Next, users apply a virtual texture to the digital model using Blender's texture mapping tools (Figure 19b). Clicking on the 'Preview' button converts the texture to the closest match realizable with the available photochromic color space (Figure 19c). To compute this preview texture, we run our optimisation algorithm on the virtual texture and compute the deactivation times $t$ for each pixel. We estimate the resulting physical color by calculating $C(t)$ and then load the newly computed texture onto the $3 \mathrm{D}$ model. The user can toggle between the virtual and the preview texture, adjusting colors as required.

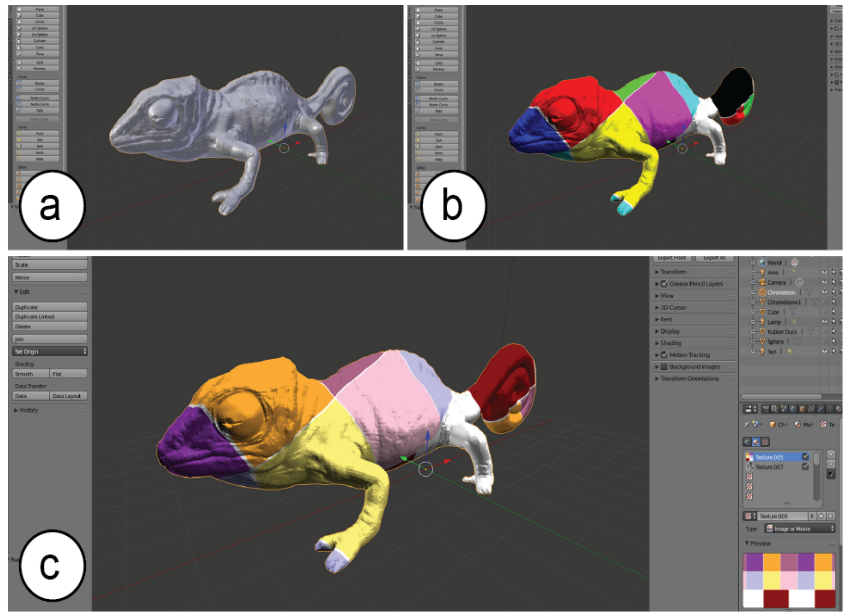

Figure 19. (a) Loading the 3D model of the object, (b) applying a virtual texture onto the 3D model, (c) computing the preview texture.

\#2 Transferring the Texture onto the 3D Object

When users hit the 'transfer texture' button, our custom python plug-in for Blender handles the projection mapping. Our system first generates four ray traced projection images by virtually rotating the $3 \mathrm{D}$ model in $90^{\circ}$ increments to reach all four sides of the object. The plug-in then sends the projection images to a Processing application (via the OSC protocol) that generates the deactivation animations. To create the deactivation animations, the Processing application converts the color values of each pixel in the projection images into deactivation times for each of the projector's R, G, B color channels. It then computes the optimal deactivation times for each pixel using our optimization algorithm. Our implemention generates this animation in 5-25 seconds per view depending on the size of the object. 
To begin the projection sequence, users hit the 'Activation' button to activate the photochromic coating on the object. The rotating platform turns $360^{\circ}$ in 10 steps $\left(36^{\circ}\right.$ increments, $1 \mathrm{~min}$ per step, $10 \mathrm{~min}$ total). Users then press the 'Projection' button to apply the computed R, G, B animations that deactivate the color channels on a per-pixel basis to create the desired color texture (Figure 20). Once the projection on one side of the object is complete, the platform rotates the object by $90^{\circ}$ and repeats this process until all the sides have processed. The total time to apply color to the object depends on the object's shape and the texture being applied (up to 45 min per side).

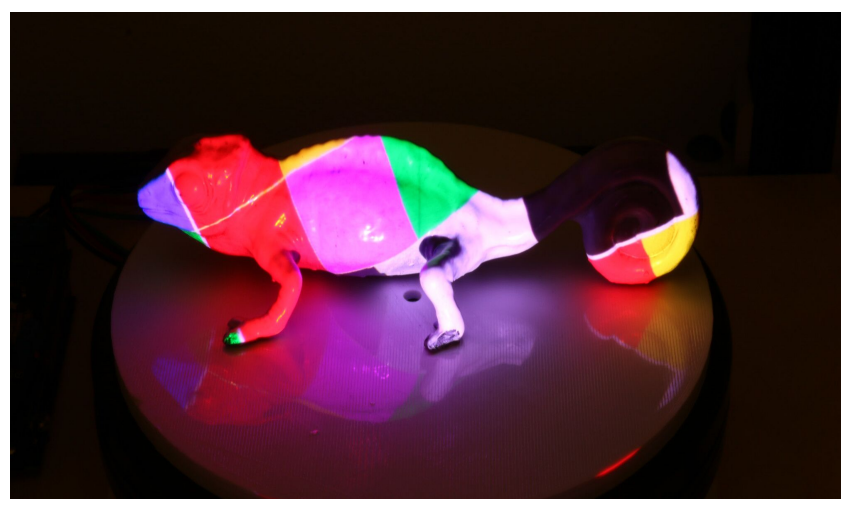

Figure 20. Projecting the deactivation colors onto the 3D object to achieve the desired texture.

Compensating for Overlap: When projecting a texture onto the object from several sides, parts of the object may be projected onto multiple times. To compensate for this, we compare the intersection points of each ray on the object to the intersection points of all other views. If a hit point is closer than the projected pixel size $(129 \mu \mathrm{m})$, we set this pixel to black, removing any projection on this area.

Compensating for Slanted Edges: The more a surface is slanted, the less light from the projector will hit each target pixel on the surface. As a result, the same deactivating time will reduce saturation more on a perpendicular surface than on a slanted surface. To compensate for this, we calculate the incident angle of each ray on the virtual object and increase the illumination time in relation to this angle (e.g. a $45^{\circ}$ angle to the projector's position increases the illumination time by a factor of 2). We limit the maximal surface angle to $65^{\circ}$ in order to limit the total illumination time.

\section{\#3 Final result}

Figure 21 shows the final result of the texture after it has been applied to the photochromic object. The texture shown took 2 hours to apply. Since the texture contains pure yellow, which requires the longest deactivation time, most other textures can be transferred significantly faster. Note that since the texture transfer is done in a black box, no decay due to external light occurs during this process. The (de)activation times could also be significantly decreased by using a stronger projector with more lumen.

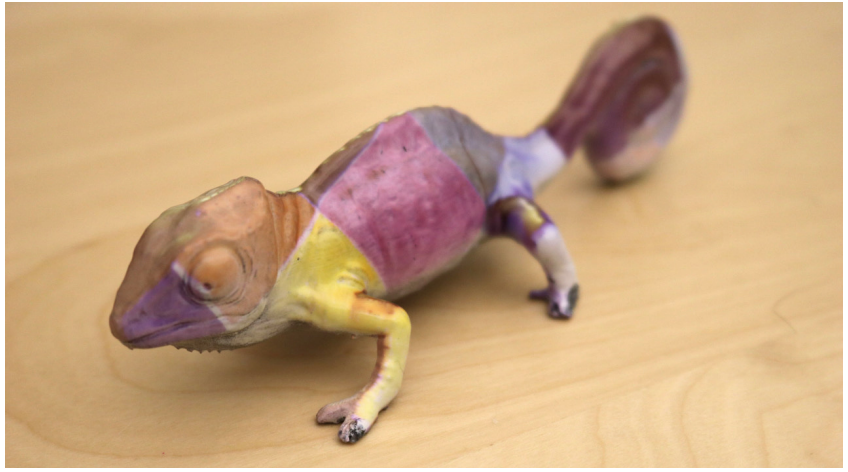

Figure 21. Final result of the texture on the object.

\section{Re-Programming (Repeat Step \#1 - \#3)}

Objects can be re-colored multiple times, simply by repeating the procedure described above, i.e. the UV light will reactivate all color channels (resulting in a black object), enabling a new texture to be applied through the projector. The zebra texture show in Figure 22 took 1 hour to apply.

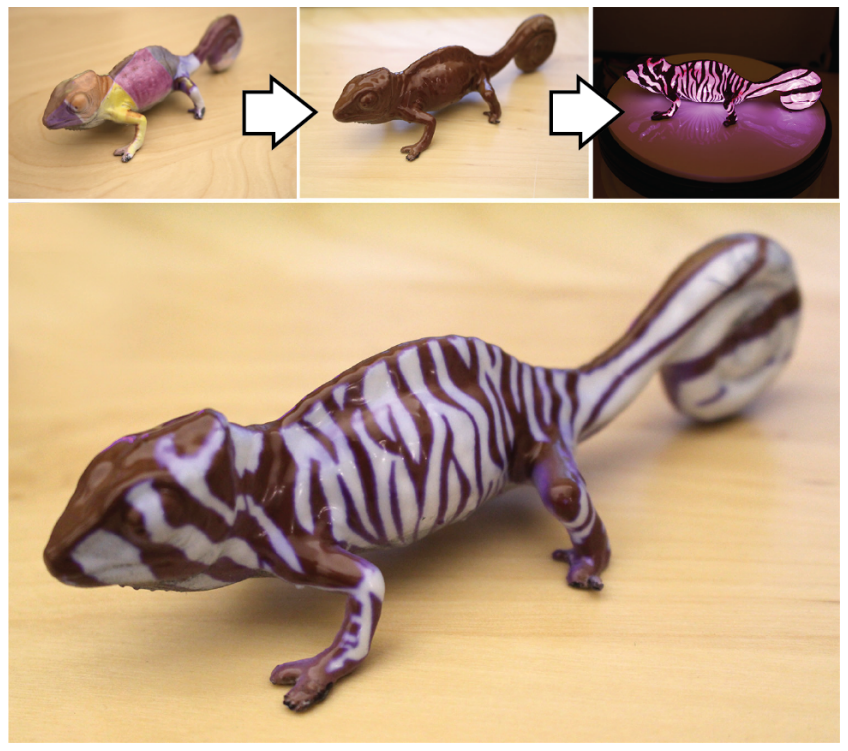

Figure 22. Re-coloring the chameleon (zebra texture) by activating with $\mathrm{UV}$ light and applying a new texture.

\section{APPLICATION SCENARIOS}

We demonstrate a range of applications that make use of our ability to reprogram textures on different materials.

\section{\#1 Day-to-Night Clothing and Adaptable Fashion}

Photochromic shoes, textiles, and accessories could enable users to alter the appearance of their outfits from day to night without the need to carry multiple items. Applying the photochromic coating to a phone case would enable users to apply and change the design or pattern of the casing on a daily basis. Here, have applied the coating to a phone case of an iPhone XR (length: $15.3 \mathrm{~cm}$, width: $7.8 \mathrm{~cm}$ ) (Figure 23). The patterns on the phone case can be re-programmed. The textures took between 24-40 minutes to apply (top: $24 \mathrm{~min}$, middle: $34 \mathrm{~min}$, bottom: $40 \mathrm{~min}$ ). 

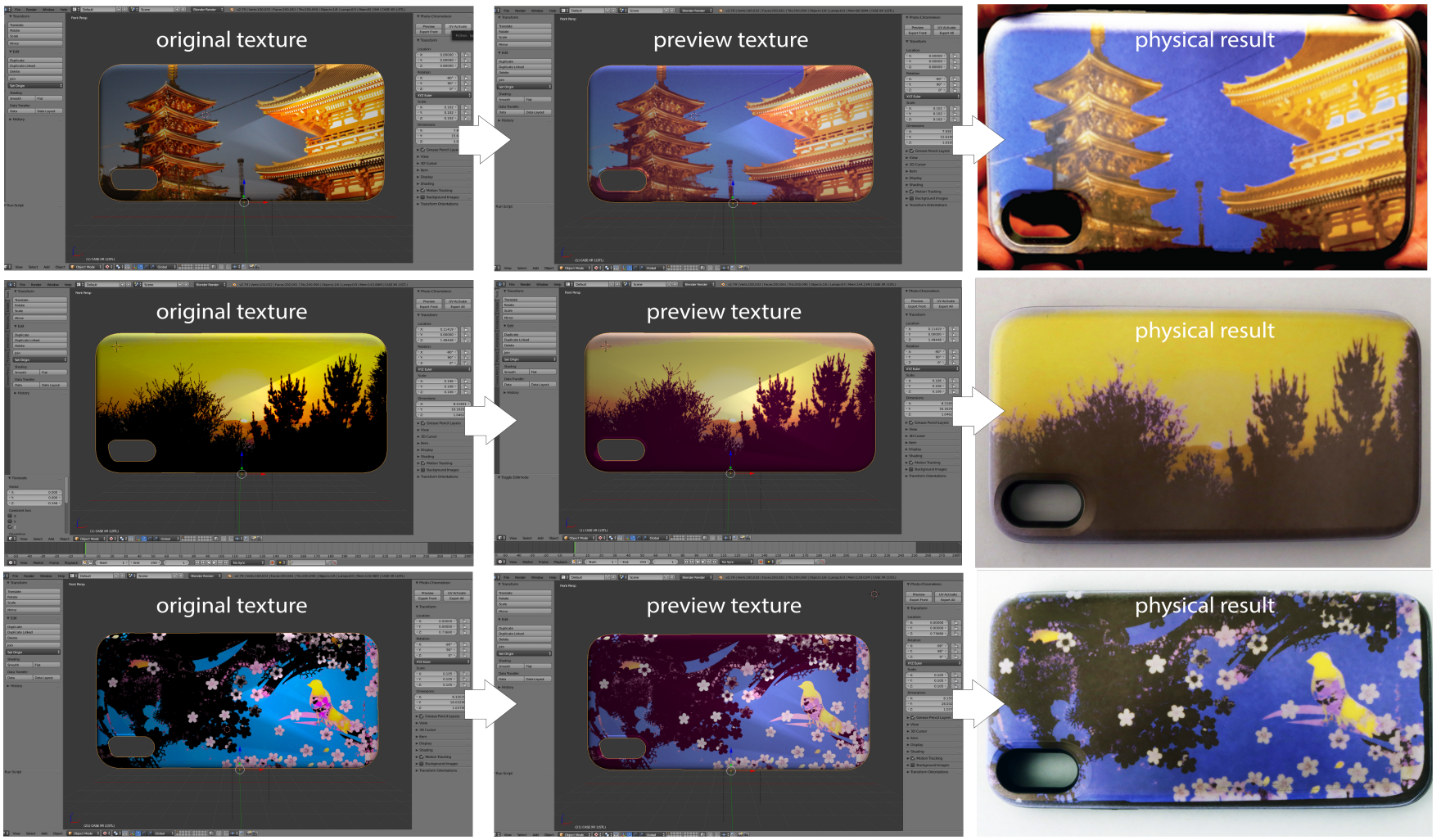

Figure 23. The same phone case re-programmed three times with different textures.

To demonstrate the application of our coating onto textiles, Figure 24 shows a women's size US 6 shoe (length: $24.5 \mathrm{~cm}$ ) coated with different textures to match different outfits; both textures took 1.5 hours to apply.

\section{\#2 Product Exploration}

In a showroom or sales scenario, a re-programmable colorchanging coating could enable potential buyers to explore different textures or patterns on a product before making a final choice. Figure 25 demonstrates this by exploring different textures for a car. To realise this vision, we sprayed the coating onto a 1/18 scale model of an Aston Martin Vanquish (length: $26 \mathrm{~cm}$ ) and projected a range of different textures. A re-programmable coating could also be used within the context of car-sharing among multiple users or to display changing advertisements on the car exterior. The textures shown took $40 \mathrm{~min}$ (butterflies) and $60 \mathrm{~min}$ (flame) to apply.

Other scenarios we envision include large-scale applications, such as entire rooms in which walls or furniture can be recolored (e.g. using a ceiling mounted UV/projector system); and dynamic physical visualizations (e.g. a 3D printed earth sphere could update during the day to show changes in weather).
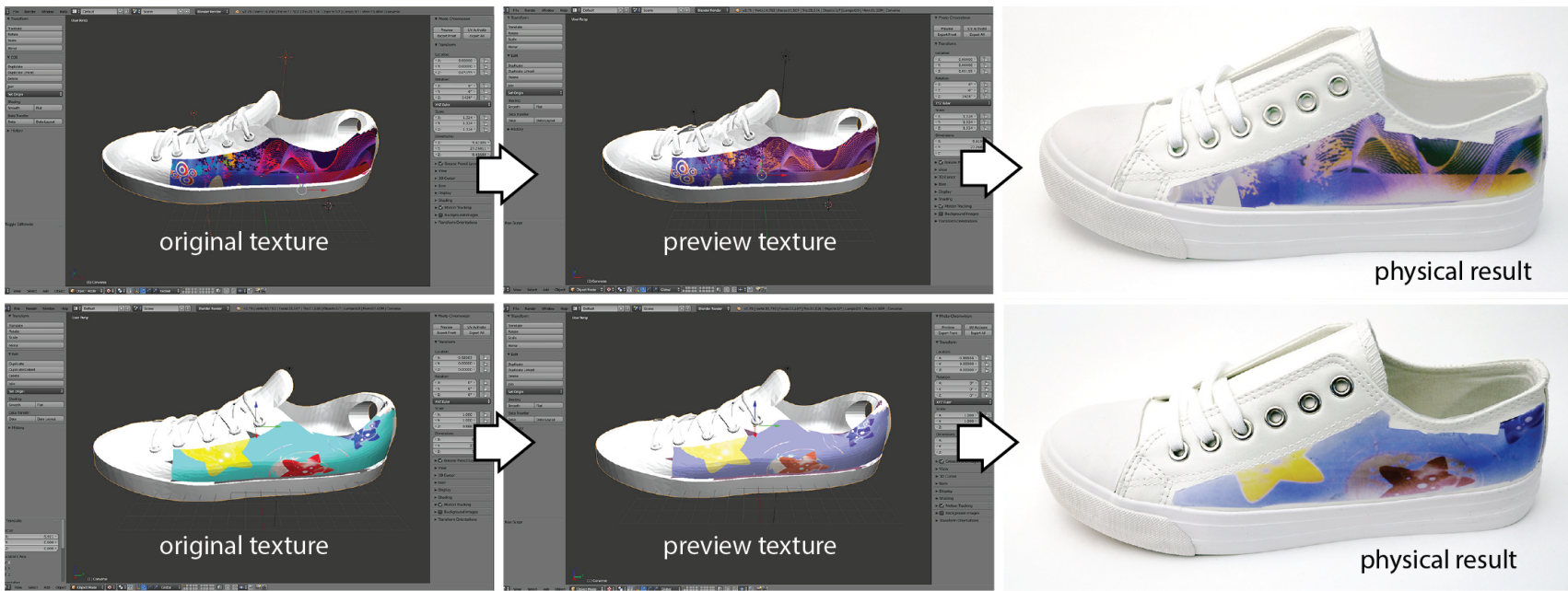

Figure 24 The same shoe with two different textures to match a user's daily outfit. 


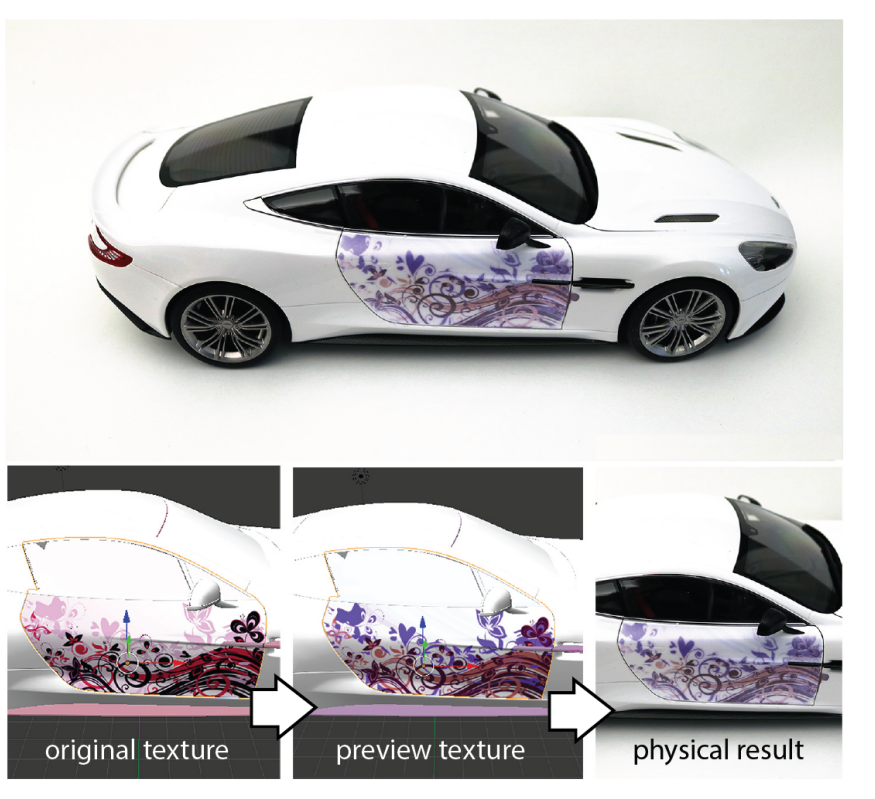

Figure 25. The same car with two different color textures.

\section{LIMITATIONS AND FUTURE WORK}

While the contributions we make in this paper extend the capabilities of prior work, there are several improvements that we will address as part of future work.

\section{Creating a Larger Color Gamut}

The photochromic dyes available on the market are currently not optimal for our approach for multiple reasons: First, among the available photochromic dyes there is no good visual match for the magenta and cyan color channels, therefore we had to approximate these color channels with the closest available dye, which significantly reduced the color gamut. The development of photochromic dyes is an on-going research field in material science. Irie et al. [12], for instance, demonstrated how to create a cyan dye that would potentially be a better candidate for our approach than Yamada Chemical's blue dye. Second, the available photochromic dyes at present signifanctly overlap in their deactivation spectra, which further reduces the available color gamut since the color channels cannot be controlled independent of one another. Photochromic dyes with narrower absorption spectra would be better suited for our purposes.

\section{Fabrication: Single Material 3D Printing (FDM, SLA):}

Our approach can turn a single-material process into a multicolor process. While we have shown applications in coating, we can also consider mixing the multi-color photochromic ink into a single roll of filament for fused deposition modelling (FDM) printing, or in resin used for stereolithography (SLA) 3D printing. This has the potential to enable multicolor prints at high resolution that are not limited by the number of extrusion heads or the single-resin process.

\section{CONCLUSION}

In this paper, we demonstrated how we can use photochromic dyes mixed into a single solution to create re-programmable multi-color textures that are made from a single material. We discussed the properties photochromic dyes

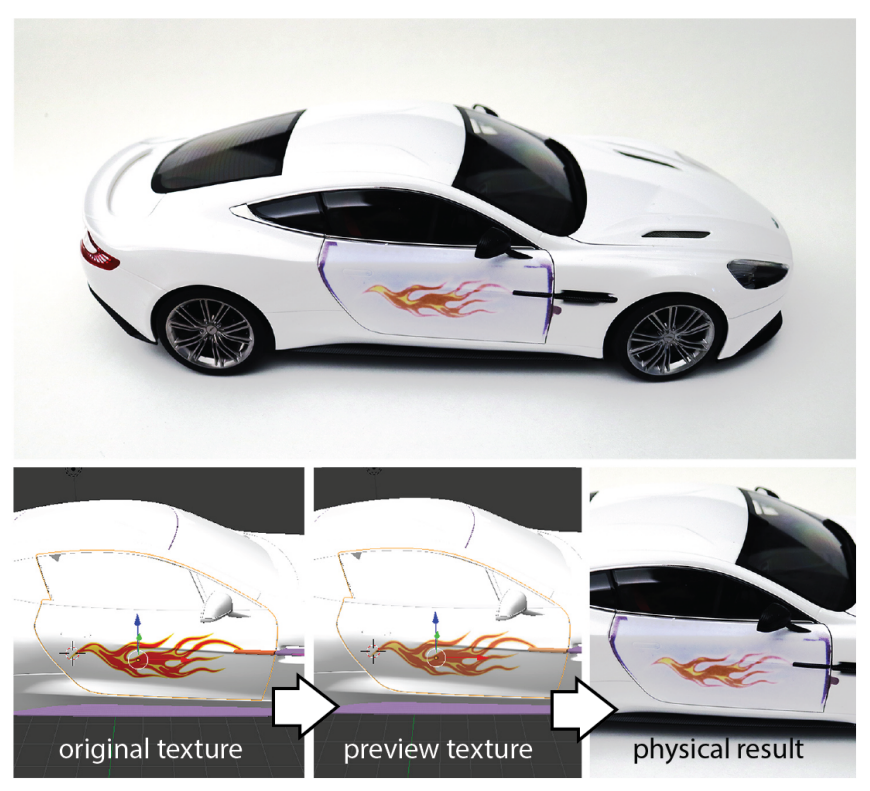

need to have in order to achieve a large color gamut (appearance as close as possible to the color channel, minimized overlap in deactivation wavelengths), demonstrated how to create a mixture solution that provides even saturation in each color channel (mixing CMY 1:1:6) and explained how the projector needs to be modified to provide deactivation wavelengths that mostly deactivate a single channel (i.e. by adding filters). Finally, we explained our algorithm to create intermediate colors and provided an end-to-end walkthrough of our system that transfers a virtual color texture onto a physical object. For future work, we plan to further increase the capabilities of our system by collaborating with material science researchers to develop improved photochromic dyes and extending our fabrication methods to $3 \mathrm{D}$ printing with filaments and resins.

\section{ACKNOWLEDGEMENTS}

The authors would like to thank the Engineering and Physical Sciences Research Council (EPSRC grant number EP/N509619/1) for partial funding of this work, Ford Motor Company for the financial support, Prof. Masahiro Irie (Rikkyo University) for sending us photochromic samples, Madeleine Laitz for help with analyzing the absorption spectrum of the photochromic dyes, Cattalyya Nuengsigkapian for help with the preview function, and Tom Buehler for support in video editing. Permission to publish was granted by Ford Motor Company.

\section{REFERENCES}

[1] Tracy Bedrosian and Randy Nelson. Timing of Light Exposure Effects Mood and Brain Circuits. In Journal of Translational Psychiatry 7, 1, 2017.

[2] Joanna Berzowska. Very Slowly Animating Textiles: Shimmering Flower. In Proceedings of $A C M$ SIGGRAPH 2004 Sketches (SIGGRAPH '04), Article 34, 2004. 
[3] Dupli-Color Matte-Finish Clear Coat https://duplicolor.com/product/paint-shop-automotive-lacquer-finish-system

[4] Marinella Ferrara, Murat Bengisu. Materials that Change Color. PoliMI SpringerBriefs in Applied Sciences and Technology, 9-60, 2014.

[5] Seth Copen Goldstein and Todd C. Mowry. Claytronics: An Instance of Programmable Matter. In Wild and Crazy Ideas Session of ASPLOS, 2004.

[6] Tomoko Hashida, Yasuaki Kakehi, and Takeshi Naemura. Photochromic Canvas Drawing With Patterned Light. In Proceedings of ACM SIGGRAPH 2010 Posters (SIGGRAPH'10), Article 26, 2010.

[7] Tomoko Hashida, Yasuaki Kakehi, and Takeshi Naemura. Photochromic Sculpture: Volumetric ColorForming Pixels. In Proceedings of ACM SIGGRAPH 2011 Emerging Technologies (SIGGRAPH '11), Article 11, 2011.

[8] Tomoko Hashida, Yasuaki Kakehi, and Takeshi Naemura. SolaColor: Space Coloration with Solar Light. In Proceedings of the fifth international conference on Tangible, embedded, and embodied interaction (TEI '11), 417-418, 2011.

[9] Ryuji Hirayama, Atsushi Shiraki, Makoto Naruse, Shinnichiro Nakamura, Hirotaka Nakayama, Takashi Kake, Tomoyoshi Shimobaba, and Tomoyoshi Ito. Optical Addressing of Multi-Colour Photochromic Material Mixture for Volumetric Display. Scientific Reports, Article 6, 2016.

[10] Masahiro Irie, Tuyoshi Fukaminato, Kenji Matsuda, and Seiya Kobatake. Photochromism of Diarylethene Molecules and Crystals: Memories, Switches, and Actuators. Chemical Reviews, 114, 24, 12174-12277, 2014.

[11] HoneyWell Ethyl Acetate https://www.fishersci.com/shop/products/ethyl-acetate-99-5-acs-reagent-honeywell-riedel-de-ha-n-7/60046011\#?keyword=Honeywell+ethyl+acetate

[12] Hsin-Liu (Cindy) Kao, Manisha Mohan, Chris Schmandt, Joseph A. Paradiso, and Katia Vega. ChromoSkin: Towards Interactive Cosmetics Using Thermochromic Pigments. In Proceedings of the 2016 CHI Conference Extended Abstracts on Human Factors in Computing Systems (CHI EA '16), 3703-3706, 2016.

[13] Hsin-Liu (Cindy) Kao, Christian Holz, Asta Roseway, Andres Calvo, and Chris Schmandt. DuoSkin: Rapidly Prototyping On-Skin User Interfaces Using SkinFriendly Materials. In Proceedings of the 2016 ACM International Symposium on Wearable Computers (ISWC'16), 16-23, 2016.
[14] Atsumi Osada, Sae Takeshita, Machi Miyahara, and Masa Inakage. KAMI CHAT. In Proceedings of the 2008 International Conference on Advances in Computer Entertainment Technology (ACE '08), 403.

[15] Parinya Punpongsanon, Xin Wen, David S. Kim, and Stefanie Mueller. ColorMod: Recoloring 3D Printed Objects using Photochromic Inks. In Proceedings of the 2018 CHI Conference on Human Factors in Computing Systems (CHI '18), Paper 213,

[16] Daniel Saakes, Masahiko Inami, Takeo Igarashi, Naoya Koizumi, and Ramesh Raskar. Shader Printer. In Proceedings of ACM SIGGRAPH 2012 Emerging Technologies (SIGGRAPH '12), Article 18, 2012.

[17] Daniel Saakes, Takahiro Tsujii, Kohei Nishimura, Tomoko Hashida, Takeshi Naemura. Photochromic Carpet: Playful Floor Canvas with Color-Changing Footprints. In Proceedings of the 11th Conference on Advances in Computer Entertainment Technology (ACE '13), 622-625, 2013.

[18] Pitchaya Sitthi-Amorn, Javier E. Ramos, Yuwang Wangy, Joyce Kwan, Justin Lan, Wenshou Wang, and Wojciech Matusik. MultiFab: A Machine Vision Assisted Platform for Multi-Material 3D Printing. In $A C M$ Transactions on Graphics 34, 4, Article 129, 2015.

[19] Kohei Tsuji, and Akira Wakita. Anabiosis: An Interactive Pictorial Art Based on Ppolychrome Paper Computing. In Proceedings of the 8th International Conference on Advances in Computer Entertainment Technology (ACE'11), Article 80, 2011.

[20] Takahiro Tsujii, Koizumi Naoya, and Takeshi Naemura. Inkantatory Paper: Dynamically Color-changing Prints with Multiple Functional Inks. In Proceedings of the 27th Annual Symposium on User Interface Software and Technology (UIST'14), 39-40, 2014.

[21] Yanan Wang, Shijian Luo, Yujia Lu, Hebo Gong, Yexing Zhou, Shuai Liu, and Preben Hansen. AnimSkin: Fabricating Epidermis with Interactive, Functional and Aesthetic Color Animation. In Proceedings of the 2017 Conference on Designing Interactive Systems (DIS '17), 397-401, 2017.

[22] Yamada Chemicals Co. Retrieved December 19, 2017 from http://ymdchem.com/

[23] Hiroki Yamada, Tomohiro Tanikawa, Kunihiro Nishimura, and Michitaka Hirose. Paint color control system with infrared photothermal conversion. In Proceedings of the 8th International Conference on Advances in Computer Entertainment Technology (ACE '11), Article 64, 2011. 